\title{
Surgical.
}

\section{THE TECHNIQUE OF OPERATIONS ON THE THYROID GLAND.}

\author{
BY A. E. MORTIMER WOOLF, F.R.c.s., \\ Surgeon to Queen Mary's Hospital for the East End, Ec.
}

THE preliminary stages for exposure of the thyroid gland are the same for all operations. The actual procedure on the gland itself depends on the disease for which the operation is undertaken, and the condition found when the gland is exposed.

I shall therefore describe the technique I usually employ, under the following headings :-

A. Preliminary steps and exposure of the gland.

B. Types of resection.

(I) Resection-enucleation.

(2) Hemi-thyroidectomy-usually for large goitres causing dyspnœa.

(3) Resection for exophthalmic goitre.

(4) Removal of intrathoracic goitre.

C. Closure of the wound.

D. Several remarks on technique.

The patient should have been rested for at least forty-eight hours before operation. In cases of exophthalmic goitre, preliminary medical treatment and rest

Preliminary

Steps and

Exposure of the

Gland. is essential, and over a longer period than is necessary for a case of ordinary goitre. I shall deal with the question of anæsthesia separately. Here, I may say, that the patient is always anæsthetized on the table. Before the aniesthetic is started, a sandbag is placed under the shoulders, and the patient's head is extended as far as is possibie. There are two reasons for for this :-

(I) In order to render the gland prominent.

(2) To ascertain beforehand how far the head may be extended without causing urgent dyspnœa. This is especially important in those cases, usually in young subjects, when a large thyroid is obstructing the trachea.

Without altering the position of the sandbag, the head is supported by pillows (which are subsequently removed before the incision is made), in order to make the patient comfortable. As the anæsthetic must be very light, the patient's legs and hands are secured to the table, in such a way that the bow securing the hands is uppermost, and can be released in a moment.

It is essential that the head be held in the middle line. This is usually done by a sister or nurse, who is instructed to hold the head by placing the palms of her hands 
along the lower margins of each mandible, which not only keeps the head steady, but serves to keep the jaws forward and prevents the tongue from falling back. This is very important, and the nurse is instructed that under no conditions is she to let go the head. Sir James Berry used to impress this on the nurse, by telling her that if an earthquake occurred, she was expected to be found dead still holding the head.

The area of operation is excluded by sterile towels. In order to isolate the neck from the head and face, a towel is folded one-third of the way up, the folded edge is placed on the chin and the head-holding nurse takes hold of the outside of the shorter leaf of the towels, holding it and jaw as described above. The long leaf is then thrown upwards which covers the nurse's fingers and the patient's face, and the area to be operated on is then completely isolated.

The usual collar incision is then made. By this is meant an incision that follows

Incision. the contour of the neck, and not one that is upturned at both extremibe concealed by a necklace.

The incision should, if possible, be placed in one of the natural creases of the neck. It should not be too high, nor too low, but a line should be aimed at, where a necklace
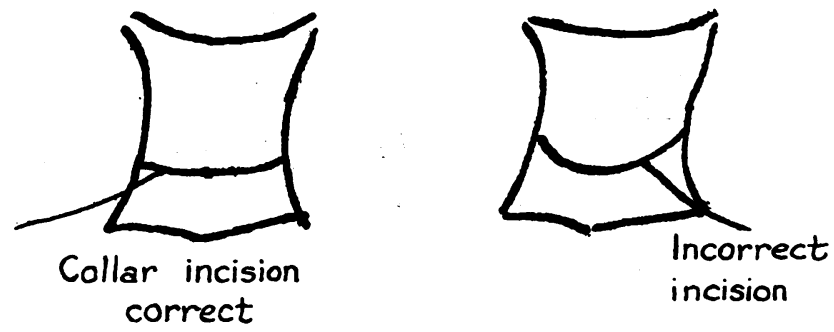

will rest in order to hide the subsequent scar. If the goitre is not too big, it is a good plan to try a necklace on the patient before operation and mark its position. The incision should be made slightly above this line, as it tends to shift downwards.

The skin and platysma should be divided at right angles. This is not quite so easy as it sounds, as if care be not taken, the platysma may be divided in more than one place and not at right angles to the skin, which may cause the scar to spread.

At this stage there may be some bleeding. Every point must be caretully caught, and no vessel allowed to ooze. The assistant should see to this while the surgeon is dissecting the upper flap. If this is not done cautiously much hæmorrhage from superficial veins will occur. The flap should be held up by a pair of toothed forceps, with such tension that a layer of areolar tissue is to be seen. With short sweeps of the scalpel the flap is raised, until the level of the thyroid cartilage is reached. The same process is done to the lower flap, but of course this flap is short.

Gauze is now placed around the skin edges, and fixed to the angles of the wound. All bleeding points are secured. As the veins for the most part run from above downwards, it is very necessary to be careful that a lateral ligature is not placed. Such a ligature is very apt to slip off perhaps after the patient has returned to bed, and a troublesome hæmatoma result. If a vein has been nicked in raising the flaps it should be 
divided completely, and terminal ligatures placed on the two ends. All bleeding points must be secured before the next stage is entered upon. If there is no urgent dyspnœa, it is sometimes a convenience to tie off all points at this stage, in order to clear the field of forceps.

The subhyoid muscular sheet, consisting of the sternohyoid and sternothyroid muscles is now exposed. Many continental surgeons advise dividing this sheet right across in the line of the incision and turning it up in toto as a flap. I

Exposure have never done this. In a very large goitre, both lobes of which may Gland. have to be removed, it might be desirable, but it seems to me unnecessary in the average case, and likely to spoil the ultimate cosmetic effect which, in a woman, cannot be neglected.

My own procedure is as follows :-

The assistant places his forefinger over the "pomum adami." This marks the line of separation between the right and left sternohyoid and sternothyroid muscles. This is not necessarily in the middle line of the neck as the larynx and trachea

Insertion of Self-retaining Retractor.

may be displaced to one side or the other. With the assistant's finger in this position the surgeon places the upper end of a Kocher's self-retaining retractor under the upper flap and the lower end is placed under the short lower flap immediately above the sternal notch. The retractor is usually inserted on the side opposite to that which is to be excised. The retractor is then stretched open and a beautiful view of the field is obtained. Many surgeons prefer to omit this retractor, but I have always found its use of much assistance as it keeps the assistant's hands out of the way and, indeed, takes the place of an extra assistant.

An incision is made in the line marked out by the retractor, and the line of cleavage between the right and left infrahyoid group of muscles, defined and divided. When incising tissues they should be held up with a forceps or with a

Separation of the

Infrahyoid Muscles. retractor. But the rat-tooth forceps should now be discarded for fear of puncturing a small vein when the surface of the gland is reached. If the tissues are held well up the most exact definition can be obtained before dividing them, and vessels can often be seen and clamped before they are cut. The incision in the mid-line should extend from the thyroid cartilage above to the sternal notch below. Iust above the sternal notch there is a very constant vessel which will require a clamp on each side of the incision.

The incision is gradually deepened and the muscles on the side to be operated on gradually retracted, until the thyroid gland is exposed. This is one of the crucial steps of the operation, and is by no means always simple or easy. If the

Importance of Accurate Definition of the Perithyroid Space.

tumour is large the muscle bundles may be stretched out and be in deep grooves in the underlying gland. Deep to the muscles there may be many layers of fascia, and although it may be thought that the gland is exposed there may still be a layer or layers of fascia over it, and at times very adherent. This is especially true in cases of exopthalmic goitre, especially if $\mathrm{X}$-ray or radium treatment has been given prior to operation. The correct layer is recognized by the branching plexus of veins over the glands, but as mentioned above it is easy to make a mistake and think that the right layer has been reached, whereas in fact a layer of fascia is still covering it. On the other hand, care must be taken not to 
puncture one of the ramifying veins on the surface of the gland. If this happens much troublesome oozing occurs which may obscure the field. Attempts to stop this by artery forceps nearly always fail, as the gland is so often friable, and the clamp only breaks more vessels and increases the oozing. The best way is to put a swab over the bleeding point and let the assistant hold it with slight pressure.

As soon as the right layer has been reached the next step is to dis-

Dislocation locate the lobe, or the tumour. This is done by inserting the finger of the Gland. into the space opened up, gently sweeping it round the posterior margin of the lobe. Before doing so, however, it should be ascertained, if the middle thyroid veins are present.

A retractor is inserted and lifted up, and the middle thyroid veins can then be identified. Often they are absent, there may be one trunk, or two or three vessels may exist. If present they should be clamped and divided, as otherwise they may be torn during the manipulations and a most troublesome hæmorrhage occur. It is at this stage that the decision must be made whether it be necessary to divide the covering muscle transversely or not. If the tumour is really big this will certainly be necessary, but generally speaking it is not necessary and with increasing experience this step is undertaken only rarely. It is because of this, that I am averse to dividing the muscles transversely at the beginning of the operation, as obviously if they can be preserved in their continuity the patient is better off. Moreover if the muscle has to be divided it should be cut high up in order to preserve its nerve supply, and also to prevent the suture line of the trunk lying directly under the scar, to which it may become adherent. Frequently it may be found, that if dislocation prove difficult owing to the size of the gland a very small transverse incision, sometimes a mere nick in the muscles, will suffice, and a complete transverse section need not be done.

The finger is then inserted, care being taken to keep in contact with the gland throughout. This finger must travel well behind, and it is in this situation if the right layer has not been entered-that damage can be done. In a large adenoparenchymatous goitre, or even in some cases of exophthalmic goitre, the posterior border of the gland may be situated very far inwards, at times touching its fellow of the opposite side and completely overlapping the œesophagus behind. Fortunately this postero-medial prolongation is always situated behind the œsophagus, and never between the o-sophagus and trachea.

The finger then delivers the lobe slowly and gradually. The assistant can often help at this stage, by exerting pressure behind the sternomastoid from without, and also with a retractor, gently pulling the tissues in a posterior direction.

As a rule, it is easier to dislocate the inferior aspect of the gland first, but if there be difficulty in getting the upper half to the surface, this should be carried out before the lower half is dislocated. It is exactly the same principle as delivering a kidney to the surface. But when all is said and done, the important factor in order to achieve this step, is to make sure that the manipulations are taking place in the correct plane.

As soon as dislocation of the lobe is satisfactorily accomplished, it should be surrounded with gauze, and the space between the lower pole and the manubrium sterni should also be lightly packed with gauze.

The surgeon has now to determine the type of operation to be carried out. 


\section{RESECTION ENUCLEATION.}

This operation is the best one to use for the removal of encapsuled tumours. The old operation of enucleation is hardly ever performed nowadays, except for very small tumours. If the tumour is of any size, enucleation may give rise to considerable hæmorrhage, and though the results were, on the whole, satisfactory, resection enucleation is the operation of choice, as the vessels are secured as a first step, and the subsequent removal of the tumour is very nearly bloodless.

The principle of the operation, first described by Sir James Berry, is to secure all the vessels feeding that part of the lobe in which the tumour is situated, and to remove with the tumour, the thin, atrophied, overlying part of the gland, which, uwing to pressure of the tumour, consists of little, if any, secreting tissue.

The first step is to secure the inferior thyroid vein. This is a large trunk, and not infrequently consists of two or three trunks. Forceps should be applied on these vessels close up to the gland. If they are placed at any distance from the gland, there is a danger of damage to the recurrent laryngeal nerve. The nerve should never be seen visually, but always mentally. If the nerve is dissected out, it will subsequently be involved in scar tissue, with resultant paralysis. There is a very definite danger to the nerve in clamping these inferior thyroid veins, if the forceps are not placed close up to the gland.

It is as well to place forceps above the ones referred to and to cut in between. The incision should extend into the substance of the lobe until the capsule of the tumour is exposed. In this situation there is a definite thickness of gland tissue to go through before the tumour capsule is reached. Forceps are then placed on all the vessels which are to be seen coursing over the tumour. These can readily be identified and the forceps should be placed as far back as possible on the thin, atrophied portion of the thyroid, care to be taken especially on the inner side, of the recurrent nerve. As soon as these forceps are in position a careful incision is made in front of them until again the capsule of the tumour is defined. By continuing this incision just in front of the clips securing the vessels, the tumour is exposed, and a cuff of thyroid tissue is differentiated. Now the enucleation part is commencing by gradually sweeping back this cuff with a pair of blunt curved scissors. A few bleeding points may have to be clipped during this process, but if the vessels have been secured as mentioned above, the bleeding is usually trivial. If it occurs it always comes from the margin of this cuff. As the enucleation proceeds the tumour is lifted up until the end stage is reached where its attachment presents as a pedicle which is clamped, cut across and the tumour removed. Bleeding points are tied, drainage provided for, and the wound closed, as described later. If the tumour is very small and the wound is quite dry, the wound may be closed without drainage. This is only rarely desirable.

(The operations for resection, the closing of the wound, and general remarks on technique will be dealt with in the next issue of the Journal.) 